\title{
Proposed Trigger Scheme for the ICAL Detector of India-based Neutrino Observatory
}

\author{
Sudeshna DASGUPTA ${ }^{* ;}$, Naba K MONDAL, Deepak SAMUEL, Mandar N SARAF, \\ Bheesette SATYANARAYANA, Suresh S UPADHYA \\ Department of High Energy Physics, Tata Institute of Fundamental Research, Mumbai 400005, \\ India \\ E-mail: sudeshnadasgupta@tifr.res.in
}

The India-based Neutrino Observatory (INO) collaboration has proposed to build a 50 kton magnetized Iron Calorimeter (ICAL) detector with the primary goal to study neutrino oscillations, employing Resistive Plate Chambers (RPCs) as active detector elements. Various aspects of a proposed trigger scheme for the ICAL detector are discussed. The associated chance trigger rates are calculated and the trigger efficiency of the scheme for the events of interest for the ICAL detector is also determined.

XI workshop on Resistive Plate Chambers and Related Detectors - RPC2012,

February 5-10, 2012

INFN Laboratori Nazionali di Frascati Italy

\footnotetext{
* Speaker.

${ }^{\dagger}$ Corresponding author.
} 


\section{Introduction}

The India-based Neutrino Observatory (INO) collaboration is planning to build a 50 kton magnetized Iron Calorimeter (ICAL) detector to detect atmospheric neutrinos and to make precision measurements of neutrino oscillation parameters, employing around 28,800 Resistive Plate Chambers (RPCs) as sensitive detector elements [1]. The ICAL detector will comprise of three modules, each of dimension $16 \mathrm{~m} \times 16 \mathrm{~m} \times 14.5 \mathrm{~m}$, housed inside a cavern with a rock cover of $1.3 \mathrm{~km}$, in order to reduce the cosmic muon background. The expected overall event rate is around $10 \mathrm{~Hz}$. The trigger scheme for such a low event-rate experiment should achieve an optimization of the detection efficiency of desired events and the chance trigger rates. It should also ensure feasibility of hardware implementation considering the vast volume of the detector module. In this paper, a trigger scheme with a distributed and hierarchical architecture, which will satisfy these criteria, is proposed for one module of the ICAL detector. Chance coincidence rates are calculated for different combinations of the trigger parameters and the trigger efficiency of the scheme is evaluated for different events of interest for the ICAL detector.

\section{The ICAL Detector and Neutrino Interactions}

The ICAL detector is envisaged as a detector for atmospheric neutrinos as well as a future end detector for a neutrino factory beam. The detector will consist of 151 horizontal layers of $56 \mathrm{~mm}$ thick low carbon iron plates interleaved with $40 \mathrm{~mm}$ gaps to house the RPC units. Table 1 contains some important parameters of the ICAL detector structure.

\begin{tabular}{|l|l|}
\hline Modules & 3 \\
\hline Module dimension & $16 \mathrm{~m} \times 16 \mathrm{~m} \times 14.5 \mathrm{~m}$ \\
\hline Detector dimension & $48 \mathrm{~m} \times 16 \mathrm{~m} \times 14.5 \mathrm{~m}$ \\
\hline Iron layers & 151 \\
\hline Iron plate thickness & $56 \mathrm{~mm}$ \\
\hline RPC layers & 150 \\
\hline Gap for RPC units & $40 \mathrm{~mm}$ \\
\hline RPC dimension & $1840 \mathrm{~mm}$ x 1915 mm x 20 mm \\
\hline RPC units/layer/module & 64 \\
\hline RPC units/module & 9600 \\
\hline Total RPC units & 28,800 \\
\hline Magnetic field & 1.3 Tesla \\
\hline
\end{tabular}

Table 1: Salient parameters of the ICAL detector structure.

Neutrino interactions in iron produce charged particles like muon and/ or hadrons. A muon typically produces a long track inside the detector, traversing many layers, while hadrons give rise to shower, confined within a few layers. The energy of the neutrino can be estimated from the momentum of the tracked muon and the hit distribution of the hadrons. The trigger criteria should be decided considering the characteristic hit pattern of such events so that the trigger system is flexible enough to cater for the events of interest and simultaneously ensures an acceptable chance trigger rate. 


\section{The Trigger Criteria}

The trigger criteria for the ICAL detector will be based on the event topology alone and is defined using three trigger parameters, namely, Multiplicity $(M)$, Layer $(N)$ and Group $(P)$. The trigger criterion is represented as $M \times N / P$, where $M$ is the number of consecutive strips on one RPC plane that have fired and $N$ is the number of layers out of a group of $P$ consecutive layers having such $M$-fold multiplicity. There will be a number of trigger criteria with different combinations of the trigger parameters corresponding to different types of physics events. Hence, the goal of the trigger system is to ensure that if an event satisfies one or more of the user-specified trigger criteria in any part of the detector, a trigger signal should be generated to initiate the data acquisition system to record the event.

\section{The Trigger Scheme}

The design of the trigger scheme follows a distributed and hierarchical architecture. The RPC, the fundamental unit of the ICAL detector, forms the base level of trigger generation, where the Level 0 and the Level 1 trigger signals are formed. The next level in the hierarchy is segment, which is a logical unit consisting of a group of RPCs, and generates the Level 2 and the Level 3 trigger signals. A number of such logical segments together constitute the detector module, which is the final level for global trigger generation.

\subsection{RPC Level}

The RPC has two orthogonal planes, namely the $X$ and the $Y$ plane, each having 64 pick-up strips, which are assumed to be two independent and identical systems.

\subsubsection{Level 0 Trigger Signal}

The signals from every 8th pick-up strip out of 64 strips on one plane of the RPC are OR-ed to generate 8 Level 0 trigger $\left(T 0_{1}, \ldots, T 0_{8}\right)$ signals, as shown below.

$$
T 0_{i}=S_{0+i}+S_{8+i}+S_{16+i}+S_{24+i}+S_{32+i}+S_{40+i}+S_{48+i}+S_{56+i}, \quad i=1,2, \ldots, 8
$$

\subsubsection{Level 1 Trigger Signal}

The Level 1 trigger $\left(T 1_{M}\right)$ signals are obtained by demanding $M$-fold coincidence of the consecutive $T 0$ signals from each RPC plane. The combinations for $T 1_{M}$ signals for $M=1,2,3,4$ are shown below.

- 1-Fold : $T 1_{1}=T 0_{1}+T 0_{2}+\ldots+T 0_{7}+T 0_{8}$

- 2-Fold : $T 1_{2}=T 0_{1} \cdot T 0_{2}+T 0_{2} \cdot T 0_{3}+\ldots+T 0_{7} \cdot T 0_{8}+T 0_{8} \cdot T 0_{1}$

- 3-Fold : $T 1_{3}=T 0_{1} \cdot T 0_{2} \cdot T 0_{3}+T 0_{2} \cdot T 0_{3} \cdot T 0_{4}+\ldots+T 0_{7} \cdot T 0_{8} \cdot T 0_{1}+T 0_{8} \cdot T 0_{1} \cdot T 0_{2}$

- 4-Fold : $T 1_{4}=T 0_{1} \cdot T 0_{2} \cdot T 0_{3} \cdot T 0_{4}+T 0_{2} \cdot T 0_{3} \cdot T 0_{4} \cdot T 0_{5}+\ldots+T 0_{7} \cdot T 0_{8} \cdot T 0_{1} \cdot T 0_{2}+T 0_{8} \cdot T 0_{1} \cdot T 0_{2} \cdot T 0_{3}$

The four Level 1 trigger signals constitute the pre-trigger signals per RPC plane and are further processed in the trigger system. 


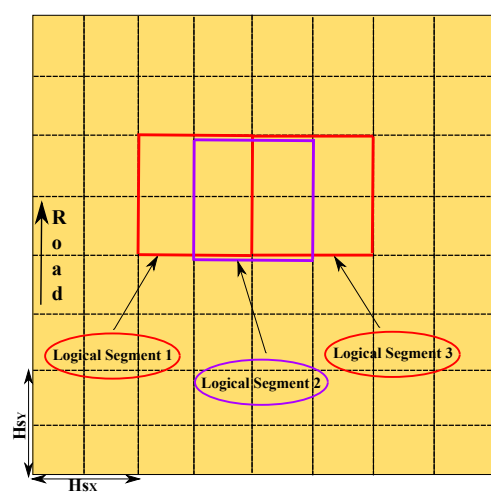

(a)

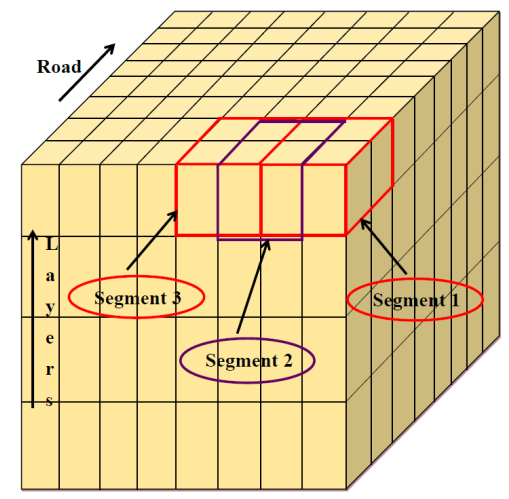

(b)

Figure 1: (a) Two-dimensional and (b) three-dimensional view of logical segmentation of the detector.

\subsection{Segment Level}

The events of interest for the ICAL detector are expected to be localized within a small portion of the total volume of the detector. Hence a distributed approach has been pursued by defining a local boundary to look for the fulfillment of the trigger criteria. The detector module is logically sub-divided into a number of identical segments, each capable of generating a local trigger, which will initiate a global trigger. The segment dimensions are chosen considering the expected hit pattern of the events, the associated chance trigger rates and the feasibility of implementation. The horizontal dimension of a segment is termed as horizontal spread $\left(H_{S}\right)$, and is interpreted as the combination of two terms, $H_{S X}$ and $H_{S Y}$, which are the number of RPCs per layer per segment along the $X$ and the $Y$ direction respectively, as shown in Fig.1(a). The vertical dimension of a segment is defined as the number of layers per segment and is termed as vertical spread $\left(V_{S}\right)$. Every two adjacent segments must have overlap between them in the horizontal and the vertical direction. Fig.1(b) shows a three-dimensional view of the logical segmentation of the detector module.

\subsubsection{Level 1 Trigger Signal}

The Level 1 trigger $\left(T 1 S_{M}\right)$ signal for a horizontal layer of the segment is the OR of the $T 1_{M}$ signals from the constituent RPCs of that layer. Thus, there will be four $T 1 S_{M}(M=1,2,3,4)$ signals per plane corresponding to each layer of a segment.

\subsubsection{Level 2 Trigger Signal}

The Level 2 trigger $\left(T 2 S_{M \mathrm{xN} / P}\right)$ signal for a segment should satisfy the trigger criterion $M \mathrm{xN} / P$ and each segment will generate one $T 2 S_{M \mathrm{XN} / P}$ signal per plane for a particular trigger citerion. The $M \times N / P$ chance rate for a $T 1 S_{M}$ rate of $R_{M} \mathrm{~Hz}$ and coincidence window of $T$ seconds is given by,

$$
r_{M \mathrm{XN} / P}=C \cdot\left(N R_{M}^{N} T^{N-1}\right) H z, \mathrm{C}=\text { combinations }
$$

\subsubsection{Level 3 Trigger Signal}

The Level 3 trigger ( $T 3 S$ ) signal for a segment is the OR of the $T 2 S_{M \times N / P}$ signals of that segment for a set of trigger criteria involving different combinations of $M$ and $N$. Hence each segment will have two Level 3 trigger signals, $T 3 S_{X}$ and $T 3 S_{Y}$, for the $X$ and the $Y$ plane respectively. 


\subsection{Module Level}

\subsubsection{Global Trigger Signal}

The global trigger $\left(G T_{X}\right.$ and $\left.G T_{Y}\right)$ signal for the $X$ and the $Y$ plane is the OR of the $T 3 S_{X}$ and the $T 3 S_{Y}$ signals respectively, from all the constituent segments of the module. The global trigger (GT) signal for the detector module is obtained as the OR of the $G T_{X}$ and the $G T_{Y}$ signals, which initiates the data acquisition system to register the event data.

\section{Chance Coincidence Rates}

The signal pick-up rate for all strips has been assumed to be identical and uniform. The average noise rate for an RPC strip of dimension $200 \mathrm{~cm} \mathrm{x} 3 \mathrm{~cm}$ is observed to be $200 \mathrm{~Hz}$ on the earth surface [2]. The RPC noise rate in the underground, as reported by the OPERA experiment, at a depth of $1.4 \mathrm{~km}$ and using RPCs of area $3.2 \mathrm{~m}^{2}$, is $17 \mathrm{~Hz} / \mathrm{m}^{2}$ [3]. The rock overburden for the INO site is similar to that of OPERA and RPCs of area $4 \mathrm{~m}^{2}$ will be used [1]. Thus, a similar figure can be assumed for the underground noise rate of the RPCs in the ICAL detector. However, the exact scenario of the radioactive background and the correlated electronic noise, which are the two main sources of RPC pick-up, are not yet known for the ICAL detector. Hence, in order to tread a safer approach, the underground noise rate for the ICAL RPCs is assumed to be 10 times that of the OPERA RPCs, i.e., $170 \mathrm{~Hz} / \mathrm{m}^{2}$. This amounts to a pick-up rate of about $10 \mathrm{~Hz}$ for an RPC strip of dimension $200 \mathrm{~cm} \times 3 \mathrm{~cm}$. The coincidence window is taken as $100 \mathrm{~ns}$ for all levels of trigger generation. Table 2 shows two different sets of trigger criteria. Each set consists of multiple trigger criteria with different combinations of $M$ and $N$, represented as $M \mathrm{x} N / P$.

\begin{tabular}{|l|l|l|l|l|}
\hline Trigger Criteria Set 1 & $1 \times 5 / 8$ & $2 \times 4 / 8$ & $3 \times 3 / 8$ & $4 \times 2 / 8$ \\
\hline Trigger Criteria Set 2 & $1 \times 4 / 8$ & $2 \times 3 / 8$ & $3 \times 3 / 8$ & \\
\hline
\end{tabular}

Table 2: Two different sets of trigger criteria.

Calculation of chance trigger rates have been done for different segment dimensions and for both sets of trigger criteria, as shown in Table 3. The GT rates are found to be the same for segments with different values of $V_{S}$ but having a fixed value of $H_{S}$. A comparison of the chance trigger rates for the trigger criteria of set 1 and set 2 implies that loosening the trigger criteria by a single layer leads to a substantial increase in the chance trigger rates. This is because $N$ comes in the exponent while calculating the $M \mathrm{x} N / P$ chance rate (Eq. 4.2) and hence a small change in $N$ causes a significant variation in the chance trigger rate.

\begin{tabular}{|c|c|c|c|c|c|c|c|}
\hline \multirow{2}{*}{$H_{S}$} & $V_{S}$ & Segment Dimension & Segments & \multicolumn{2}{|c|}{ Rates for Trigger Criteria Set 1 } & Rates for Trigger Criteria Set 2 \\
\cline { 5 - 8 } & & & Surface $(\mathrm{Hz})$ & Underground $(\mathrm{Hz})$ & Surface $(\mathrm{Hz})$ & Underground $(\mathrm{Hz})$ \\
\hline $4(2 \times 2)$ & 40 & $4 \mathrm{~m} \times 4 \mathrm{~m} \times 4 \mathrm{~m}$ & 196 & 87 & $2.7 \times 10^{-5}$ & $1.4 \times 10^{4}$ & $8.5 \times 10^{-2}$ \\
\hline $9(3 \times 3)$ & 60 & $6 \mathrm{~m} \times 6 \mathrm{~m} \times 6 \mathrm{~m}$ & 108 & $3.7 \times 10^{3}$ & $1.1 \times 10^{-3}$ & $2.6 \times 10^{5}$ & 1.6 \\
\hline $16(4 \times 4)$ & 80 & $8 \mathrm{~m} \times 8 \mathrm{~m} \times 8 \mathrm{~m}$ & 50 & $4.5 \times 10^{4}$ & $1.4 \times 10^{-2}$ & $1.8 \times 10^{6}$ & 11.1 \\
\hline
\end{tabular}

Table 3: Chance trigger rates on the surface and underground for different sets of trigger criteria and different segment dimensions. 


\section{Trigger Simulation Framework}

A simulation framework has been developed in order to evaluate the performance of the proposed trigger scheme by determining the trigger efficiency of the scheme for different events of interest for the ICAL detector. The simulation package based on GEANT4 [4] and ROOT, developed in order to study the physics potential of the ICAL detector, has its own Monte-Carlo event generator using which muon events have been generated while Nuance [5] has been used for generating neutrino events, over a wide energy range and different incident directions. The event vertices are randomly distributed over the fiducial volume of the detector. These events have been simulated using the INO-ICAL simulation code and the output of the digitization stage, which provides hit position information in the detector in the same format as that which will be available from the detector data acquisition system, has been used as the input for the current analysis.

\subsection{Results}

The algorithm to determine whether an event has satisfied the trigger criteria has been developed in compliance with the hierarchy of the proposed trigger scheme. The trigger efficiency $\eta$ is defined as,

$$
\eta=\frac{N_{E}}{N_{T}}
$$

where $N_{E}$ is the number of events satisfying the trigger criteria and $N_{T}$ is the total number of events, and is determined for the trigger criteria Set 1 (Table 2) and the segment size of $4 \mathrm{~m} \mathrm{x} 4 \mathrm{~m} \mathrm{x} 4 \mathrm{~m}$.

\subsubsection{Muon Events}

Muon events in the energy range of 1 to $10 \mathrm{GeV}$ with the incident direction varying from $0^{\circ}$ to $90^{\circ}$ have been analyzed. Fig.2(a) shows the variation of trigger efficiency with muon energy for incident directions $0^{\circ}, 20^{\circ}, 40^{\circ}, 60^{\circ}$ and $80^{\circ}$. The efficiency increases with the increase in energy because a particle with higher energy traverses a longer path through the detector and has a greater probability of satisfying the trigger criteria. Since the particles incident at a large angle traverse lesser number of layers, their chance of satisfying the trigger criteria is lower and hence the efficiency deteriorates even at higher energy for large angles of incidence. The variation of trigger efficiency as a function of different combinations of the trigger parameters $M$ and $N$ is shown in Fig.2(b). It is evident that in case of muon events, the efficiency is dominated by the 1-Fold and the 2-Fold criteria. The trigger efficiency is found to remain almost unchanged with variations in other trigger parameters like $P, H_{S X}, H_{S Y}$ and $V_{S}$ for muon events as well as neutrino events.

\subsubsection{Charged Current Neutrino Events}

Charged current neutrino events in the energy range of 1 to $10 \mathrm{GeV}$ with the incident direction varying from $0^{\circ}$ to $90^{\circ}$ have been studied. Fig.3(a) shows trigger efficiency varying as a function of neutrino energy for incident directions $0^{\circ}, 20^{\circ}, 40^{\circ}, 60^{\circ}$ and $80^{\circ}$. The efficiency clearly goes up with the increase in energy and comes down for larger incident angles. The variation of trigger efficiency for different combinations of the trigger parameters $M$ and $N$ is shown in Fig.3(b). The contribution of trigger criteria with $M>2$ seems to be significant for charged current neutrino events in comparison with muon events (Fig.2(b)). This is due to the hadron shower produced as a result of the interaction which satisfies the 3-Fold and the 4-Fold trigger criteria. 


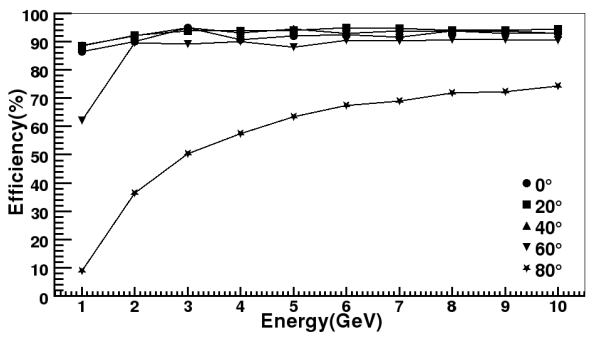

(a)

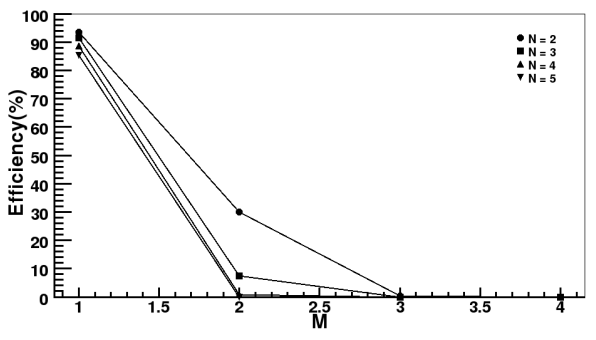

(b)

Figure 2: (a) Trigger efficiency vs. energy for muon events of energy 1 to $10 \mathrm{GeV}$ and (b) trigger efficiency vs. trigger parameters $(M, N)$ for muon events of energy $1 \mathrm{GeV}$ and incident direction $10^{\circ}$.

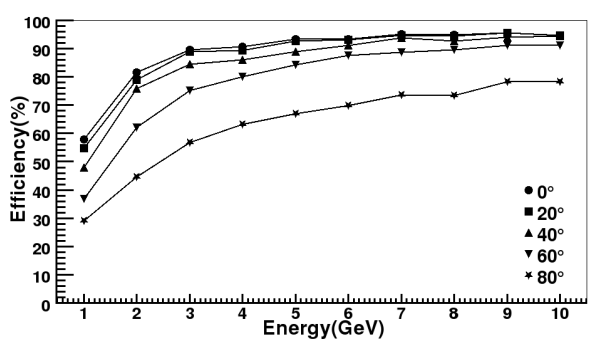

(a)

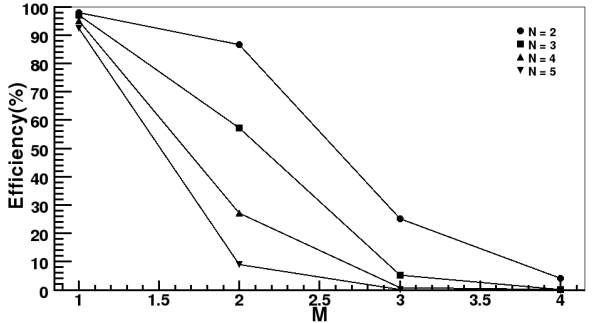

(b)

Figure 3: (a) Trigger efficiency vs. energy for charged current neutrino events of energy 1 to $10 \mathrm{GeV}$ and (b) trigger efficiency vs. trigger parameters $(M, N)$ for charged current neutrino events of energy $5 \mathrm{GeV}$ and incident direction $10^{\circ}$.

\section{Conclusion}

Various features of a proposed trigger scheme, which will form an integral component of the data acquisition system for the ICAL detector, have been presented here. The associated chance trigger rates are found to be acceptable for an optimal combination of the trigger parameters. The simulation results provide a good assessment of the detection efficiency of the trigger scheme for the events of interest for the ICAL detector and also help to understand the nature of variation of trigger efficiency as a function of the trigger parameters. The proposed scheme thus been validated, has helped us gain confidence to proceed towards contemplating the implementation of the same.

\section{References}

[1] INO Project Report, vol.1, http: //www. ino.tifr.res.in/ino/, (2006).

[2] M. Bhuyan, Development of $2 m \times 2 m$ size glass RPCs for INO, doi:10.1016/j.nima.2010.09.087, Nucl. Instr. and Meth. A., (2010).

[3] A. Garfagnini, The OPERA muon spectrometers, Nucl. Instr. and Meth. A, 572(1):177-180, (2007).

[4] J. Allison, Geant4-a simulation toolkit, Nucl. Instr. and Meth. A, 506(3):250-303, (2003).

[5] D. Casper, The nuance Neutrino Physics Simulation and the Future, Nucl. Phys. Proc. Suppl., 112:161-170, (2002). 